\title{
LA VIVIENDA PROPIA COMO PRÁCTICA INTEGRADA EN TECNOLOGÍA DEL CICLO INTRODUCTORIO FAUD, UNMDP
}

Eje 1: Innovación en sistemas constructivos/estructurales

\author{
Goity Gilma Beatriz ${ }^{1}$
}

Fenoglio Carlos Eduardo²

\section{Colaboradores: Oteiza Nicolás Hernán; Villén María Tatiana; Terra Loredo Agustín \\ Lautaro $^{3}$}

\author{
${ }^{1}$ Introducción a las Construcciones, Matemática, Centro de Estudios de Diseño, Facultad de Arquitectura, \\ Urbanismo y Diseño, UNMDP, Mar del Plata, Argentina, gilma.goity@gmail.com \\ 2 Introducción a las Construcciones, CED, FAUD, UNMDP, Mar del Plata, Argentina, cafenoglio@gmail.com \\ 3 Introducción a las Construcciones, CED, FAUD, UNMDP, Mar del Plata, Argentina,
}

\section{RESUMEN}

Emplazado en Introducción a las Construcciones, materia del área Tecnológico-Constructiva, correspondiente al primer año de la carrera de Arquitectura, Facultad de Arquitectura, Urbanismo y Diseño de la Universidad Nacional de Mar del Plata, el trabajo describe la experiencia pedagógica de la integrante tecnológica en arquitectura. Fundamentando la propuesta en la reflexión de la arquitectura como abrigo a través de la problemática de su materialización y de su funcionamiento, resaltando la relación diseño, proyecto y tecnología. El estudiante toma conciencia que no se puede pensar en dibujar sin el correlato de la realidad concreta, donde cada línea sobre el papel representa un material diferente, con sus propiedades, dimensiones, tecnología de producción, de usos y costos, no pudiendo disociar diseñar de construir.

Los contenidos de la asignatura se desarrollan en Unidades Temáticas que constituyen marcos de referencia para planificar y organizar experiencias de aprendizaje alrededor de áreas comunes de conocimiento, proporcionan una organización y estructura. Agrupan contenidos y obtienen la denominación de aquel que se considera su rasgo principal. Para su aprendizaje se propone una secuencia didáctica que permite alcanzar el contenido central de ese tema y cumplir los objetivos. Es desarrollada en espacio de tiempo con actividades prácticas de distinto tipo. Cada unidad selecciona una serie de contenidos que corresponden a un recorte del campo disciplinar, establece actividades en una metodología de acercamiento con su verificación y evaluación parcial y final.

El trabajo sobre la vivienda propia representa la síntesis de los objetivos formulados en cada unidad temática durante todo el proceso anual. A través de la relación directa y sistémica con su vivienda, el estudiante realiza la síntesis entre: observación y contacto sensible con la realidad, la simbolización como modo de expresión, y la modelización a través de cálculos que simulen hechos reales. 
Su realización comprende tareas de medición, relevamiento, simbolización, interpretación, verificación, análisis, síntesis, modelización, investigación. Se divide en 6 partes: 1. Acercamiento a la vivienda, relevamiento sensorial y descriptivo; 2. Comportamiento del edificio frente a las distintas solicitaciones, donde se analizan las propiedades mecánicas, térmicas, higrotérmicas, hidrófugas y acústicas; 3. Documentación gráfica de la vivienda; 4. Instalaciones; 5 . Identificar y analizar materiales; 6. Investigar un material. El trabajo también se propone como un lugar de clarificación de las necesidades del área, sobre la enseñanza de la tecnología, en un estudio que sustente decisiones acertadas en los procesos pedagógicos para aprehender una realidad material de difícil síntesis.

\section{PALABRAS CLAVES: TECNOLOGÍA - PEDAGOGIA - DISEÑO - INTEGRACIÓN}

\section{INTRODUCCIÓN}

Introducción a las Construcciones, ocupa el espacio pedagógico de primer año de la carrera de Arquitectura de la Facultad de Arquitectura, Urbanismo y Diseño (FAUD), de la Universidad Nacional de Mar del Plata (UNMDP), se ubica en el área Tecnológico-constructiva, sub área Construcciones. Su finalidad es iniciar al estudiante en el conocimiento específico del Hacer Arquitectónico, sentando las bases teórico-prácticas que evolucionaran a lo largo de la carrera.

El presente trabajo refiere a una producción parcial de las actividades desarrolladas en el año por la cátedra. La vivienda propia, como práctica integradora, alude a las conclusiones del sujeto en relación al aprendizaje abordado en un ciclo lectivo.

El docente, moderador disciplinar, organizador de la información, mediador, investigador, transmisor de cultura, estimulador y facilitador será el conductor del proceso de enseñanza de saberes orientando al estudiante. Dice Paulo Freire1, "Un profesor que piensa acertadamente deja visualizar a los educandos que una de las bellezas de nuestra manera de estar en el mundo y con el mundo, como seres históricos, es la capacidad de conocer el mundo al intervenir en él".

En este proceso didáctico aprender-enseñar-orientar, todas las partes son importantes. Los métodos, técnicas y recursos utilizados serán indispensables en el sistema. Enseñar exige rigor metódico, investigación, respeto a los saberes de los educandos, crítica y reflexión hacia la propia enseñanza. Adhiriendo a Freire1, en cuanto enseñar no es transferir conocimientos, sino crear las posibilidades de su producción, o de su construcción, por ello exige buen juicio, convicción de que el cambio es posible, seguridad, compromiso, competencia profesional y generosidad.

La propuesta articula y ordena un sistema de complejidades crecientes, donde el estudiante aprende mediante el ejercicio cíclico de la resolución de problemas sistémicos y específicos. Adquiriendo los conocimientos tecnológicos de modo integrado y totalizador al proyecto arquitectónico, mediante la observación, la formulación de interrogantes y la práctica que traduce en nuevos conceptos a ser aprendidos y el espacio de la teoría como insumo necesario para la formación disciplinar.

El estudiante forma su propio entramado cultural, el del campo específico de competencias, más o menos generalizada y en el sentido más amplio, intentando organizar entre sí las informaciones 
desde el día de su nacimiento. La educación debe tender a colaborar a esa organización, tanto en la escuela primaria, la secundaria y más tarde en la universitaria.

Por ello desde el comienzo formativo en la universidad, en sus experiencias proyectuales iniciales, el estudiante debe vincular diferentes aspectos en la disciplina. Como dice Quaroni1 (1980), al reformular el triángulo vitruviano, en la construcción de toda obra de arquitectura hay que tener en cuenta que la concepción de la misma es el resultado de los contenidos sociales y de las razones institucionales por las que una determinada sociedad o poder, requiere de ella. Esas razones humanas deben ser la base de toda buena proyectación. La estructura espacial considerada la más idónea para responder a la demanda social, deberá concebirse en términos constructivotecnológicos, es decir con el empleo de materiales adecuados; ser resistente estáticamente; y poder protegerse del calor, del frío, del ruido, del sol, la humedad, los ojos y manos indiscretas. Finalmente ambas operaciones hay que hacerlas sirviéndose de las capacidades de control proporcionado por la cultura arquitectónica que tiende a que ambas anulen sus incompatibilidades recíprocas para transformarse en su resultante, la arquitectura.

\section{DESARROLLO}

\section{LA VIVIENDA PROPIA COMO PRÁCTICA INTEGRADORA}

Son objetivos de la asignatura que al término del curso el estudiante haya adquirido el contacto con los materiales del medio real y sus propiedades, para un uso adecuado y racional, a fin de que en los niveles siguientes de construcciones, avance progresivamente en el desarrollo de sistemas constructivos tradicionales y no tradicionales.

Los contenidos de la asignatura se desarrollan en Unidades Temáticas que constituyen marcos de referencia para planificar y organizar experiencias de aprendizaje

Cada unidad selecciona una serie de contenidos que corresponden a un recorte del campo disciplinar, establece actividades en una metodología de acercamiento con su verificación y evaluación parcial y final. Estructurándose teniendo en cuenta el currículo institucional, la carga horaria, estrategia de cátedra y objetivos institucionales, generales y particulares de la asignatura, ellos están claramente definidos e interactúan permanentemente.

Las Unidades Temáticas se dividen en dos ejes, uno por cuatrimestre. El primero de ellos se refiere a las solicitaciones del entorno y la ecología; y las propiedades de los materiales (física de la construcción). Se destacan: Peso Específico y fuerza de la gravedad; solicitaciones mecánicas, cómo se deforman las estructuras; solicitaciones térmicas, ahorro de energía, clima, cambios térmicos en la construcción; solicitaciones higrotérmicas, condensaciones; solicitaciones hidrófugas, el control de humedad; solicitaciones acústicas, el control del ruido, aislación y absorción; propiedades eléctricas, conceptos básicos para instalaciones eléctricas; y propiedades de los fluidos, conceptos básicos para las instalaciones sanitarias. Todas dictadas en el primer cuatrimestre.

El segundo eje se refiere a los Materiales de construcción, respuestas, comportamientos ante las solicitaciones, patologías, comercialización y producción. Se destacan: aglomerantes y aglomerados; pétreos naturales; cerámicos, tierras crudas y cocidas; maderas, naturales, implantadas, industrializadas; vidrios; plásticos, termoplásticos y termoestables; los materiales ante las solicitaciones, comportamientos y requisitos de uso; dictados en el segundo cuatrimestre. 
Los recursos pedagógicos y la metodología secuencial (figura 1) consisten en Cuestionarios, Conferencias, Ejercicios de simulación, Dinámica de Grupos, Investigación y Trabajo Práctico Único.

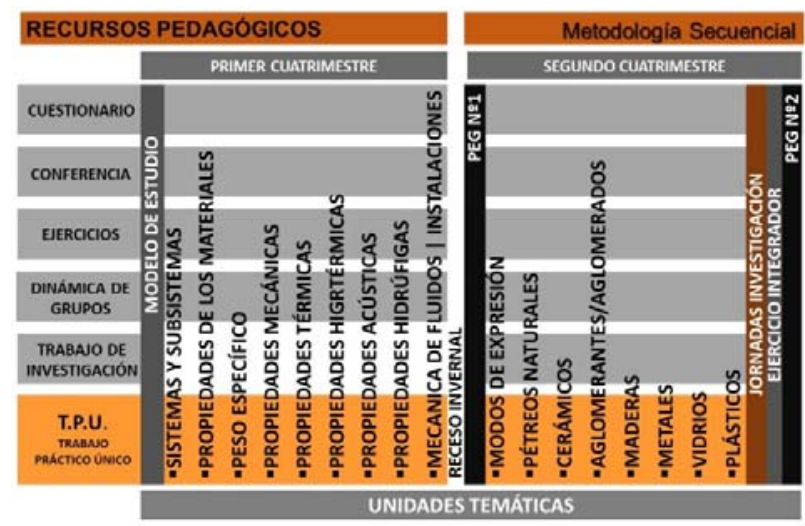

Fig. 1: cuadro Recursos Pedagógicos y Metodología Secuencial

\section{TRABAJO PRÁCTICO ÚNICO (TPU)}

En paralelo a las actividades planteadas, se realiza el trabajo sobre la vivienda propia como práctica integrada en tecnología, llamado en la cátedra Trabajo Práctico Único (TPU).

Es propósito del trabajo lograr los mecanismos necesarios para verificar y unificar los saberes desarrollados secuencialmente en el curso a través de la realidad en su propia vivienda.

Su objetivo es intentar que el estudiante a través de la relación directa y sistémica con su Vivienda, realice la síntesis entre: observación y contacto sensible con la Realidad, simbolice ésta como modo de expresión, Simbolización, (importante la herramienta del dibujo y la incorporación de la informática) y modele esa realidad a través de cálculos que simulen hechos reales, Modelización. Comprende tareas de medición, relevamiento, simbolización, interpretación, verificación, análisis, síntesis, modelización, investigación. Este trabajo intenta que el estudiante realice una síntesis integradora de los contenidos y objetivos propuestos por la Cátedra, y sirva de verificación.

Se desarrollan, los objetivos y contenidos incorporados en las Unidades Temáticas. Toma como base de ejecución los cuestionarios, las conferencias, ejercitaciones, dinámicas de grupos y bibliografía general y complementaria.

Su tiempo de ejecución es de un año lectivo. La presentación es en carpeta tamaño A4 u oficio, con carátula identificadora del estudiante con nombre, apellido, ciclo lectivo, foto $4 \times 4$. Cada lámina, plano o informe deberá contener un rótulo identificando Unidad Temática, sub-tema, escala (de ser necesario), datos personales y espacio para visado por parte del docente. La realización y presentación es individual, con representación libre, siendo su única condición que sea legible y en original. 
El TPU está dividido en seis (6) partes. Figura 2.

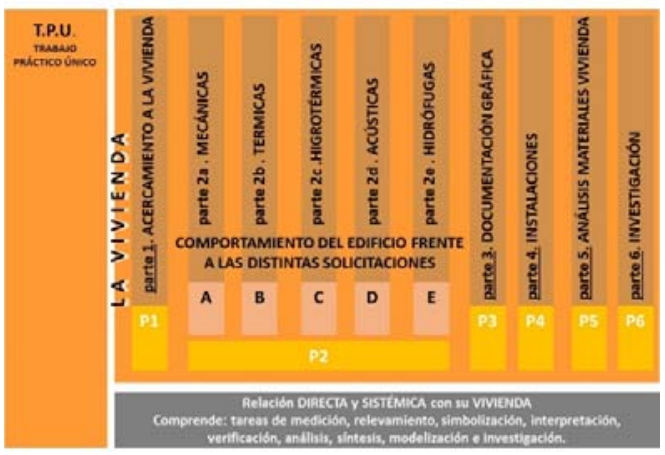

Fig. 2: Partes del TPU

\section{EL TRABAJO PRÁCTICO ÚNICO COMO PRÁCTICA TRONCAL EN IC.}

El trabajo da inicio con el Acercamiento a la vivienda, relevamiento sensorial y descripción, Parte 1. Unidades Temáticas intervinientes: Modos de Expresión e indirectamente Propiedades Térmicas (interpretación del asoleamiento). Propiedades acústicas (influencia de ruidos).

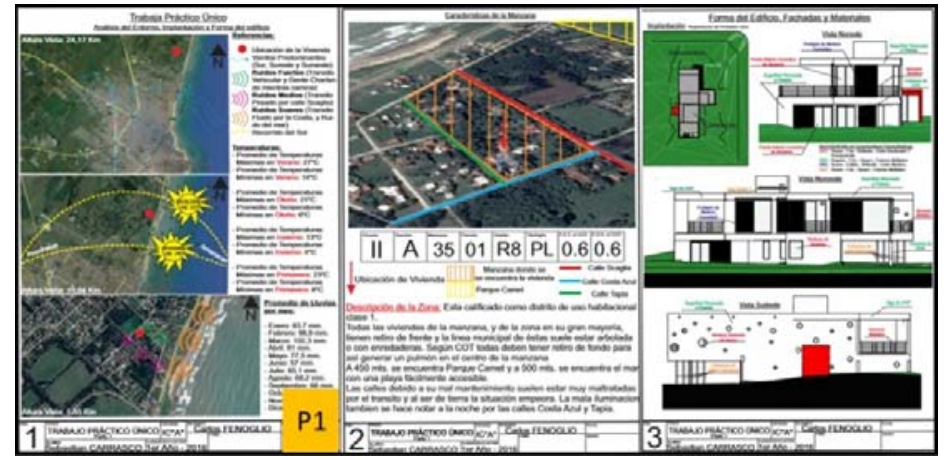

Fig. 3. Parte 1 TPU. Acercamiento a la vivienda

Se identifican, a partir de un gráfico en planta de la manzana y su entorno: la denominación y recorrido de las calles, el relevamiento del tránsito peatonal y vehicular, la distinción de usos (comercial-residencial-industrial-educacional) y los espacios libres y construidos. En un esquema del terreno se establece la implantación de la vivienda, su forma, ubicación de la Línea Municipal, los retiros de frente, las medidas del terreno, y tipo de cubierta de techo: plana o con pendiente. Se acompaña el análisis en forma escrita, gráfica o con planillas, reconociendo la influencia de factores climáticos de la zona, tales como vientos dominantes, lluvias, cercanías al mar y temperaturas máximas y mínimas en las distintas estaciones del año. Mediante esquemas en planta de la vivienda se registra la incidencia del sol en los momentos de la mañana, el mediodía y la tarde. Finalmente, por medio de una vista o fotografías, se identifican los materiales que reconozca de la envolvente. Figura 3.

La parte 2 del trabajo denominada Comportamiento del edificio frente a las distintas solicitaciones, se subdivide en 2a. Solicitaciones Mecánicas, referenciando la Unidad Temática del mismo nombre. 2b-Solicitaciones Térmicas, participa la Unidad Temática Propiedades Térmicas; 2c- Solicitaciones Higrotérmicas, intervienen las Unidades Temáticas de Propiedades Térmicas e Higrotérmicas; 2dSolicitaciones Hidrófugas, interpone la Unidad Temática Propiedades Hidrófugas; 2e. Solicitaciones 
Acústicas con su correlato de Unidad temática. En cada una de las partes, el estudiante realiza un análisis crítico de su vivienda con relación a las solicitaciones y plantea alternativas en busca de soluciones.

La parte 2A afectada a las Propiedades Mecánicas (Figura 4). A partir de la planta de su vivienda, el estudiante identifica las partes estructurales, clasificando las mismas en portantes, independientes o mixtas. En un esquema en corte señala el recorrido de cargas hasta el suelo firme, las deformaciones de cada una de las partes y a qué esfuerzos simples están sometidas (traccióncompresión-flexión-corte). El análisis culmina con un cálculo de los pesos de las partes de un sector de la vivienda hasta el suelo firme, para luego calcular la tensión y compararla con la tensión admisible de la zona. En este caso se pregunta ¿qué pasaría? si la tensión supera la admisible y se formularán respuestas. Es objetivo de esta parte del trabajo que el estudiante comprenda la importancia que el sub-sistema estructural ocupa en una obra de arquitectura; que interprete las nociones de equilibrio, recorrido y tipo de cargas; identifique los estados básicos de tensión y diferencie estructuras portantes, independientes y mixtas.

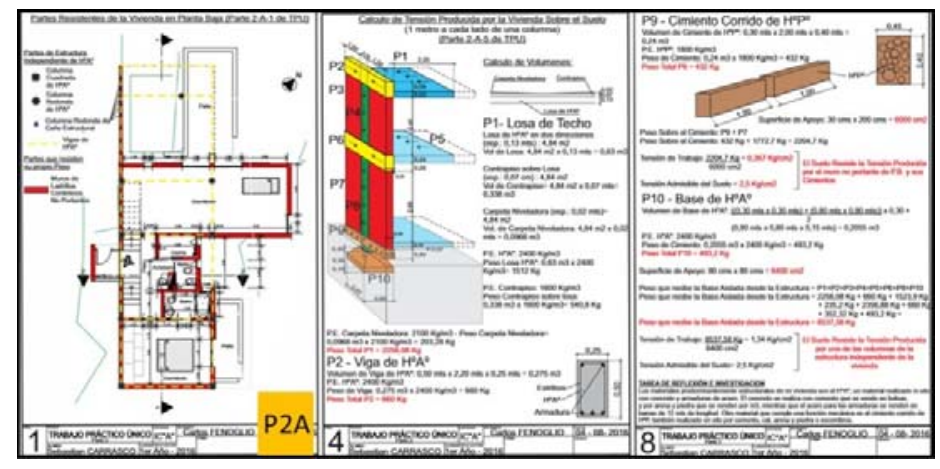

Fig. 4: Parte 2A Análisis de las Propiedades mecánica

Para el análisis de las Solicitaciones Térmicas, Parte 2B, el estudiante analiza, en planta y corte, dónde hay perdida de calor, para desarrollar cálculos de Resistencia Térmica $(R)$ y Transmitancia Térmica $(K)$, acompañado con gráficos, de cada uno de los elementos que conforman la envolvente de su vivienda (Techo-Muros-Aberturas-Piso). Luego se ejecutan cálculos de Flujo Térmico (Q), apoyados con gráficos de las distintas superficies intervinientes y el análisis de la diferencia de temperatura, para culminar con un estudio integral de las pérdidas de kilocaloría sobre hora teniendo en cuenta el K, las superficies y las pérdidas por hora en cada parte. La práctica en este segmento del TPU culmina con una propuesta de mejora, teniendo en cuenta las proporciones entre opacovidriado, los $\mathrm{K}$ en relación a los $\mathrm{K}$ admisibles y las superficies expuestas. Figura 5 . Será objetivo de estas propiedades, que el estudiante reconozca el ahorro energético como condicionante de diseño, en sus distintas escalas, particularmente en el acondicionamiento térmico de la envolvente. 


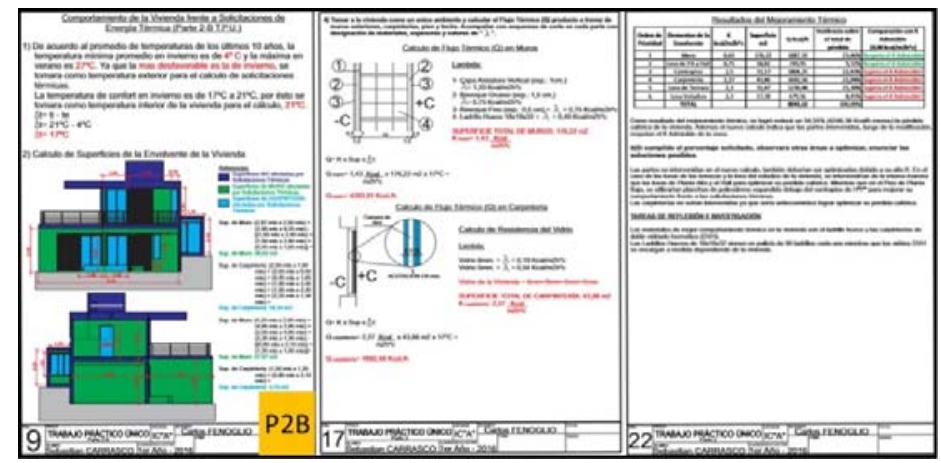

Fig. 5. Parte 2B. Propiedades Térmicas

Teniendo en cuenta los conceptos higrotérmicos, en la parte $2 \mathrm{C}$, el estudiante indaga en cada componente de la envolvente, a través de un corte, planta y/o vista, la posibilidad de condensación del vapor de agua (Permanente-Transitoria-Superficial-Intersticial), las posibles patologías y soluciones. Indica las condiciones adversas en la materialidad del edificio y su orientación. Identifica los puentes térmicos y sus posibles soluciones. Figura 6, parte P2C. Como objetivo el estudiante debe conceptualizar la condensación como un fenómeno perjudicial para el confort de su vivienda y entender que a partir de un buen diseño bioclimático dará respuesta al ahorro energético.

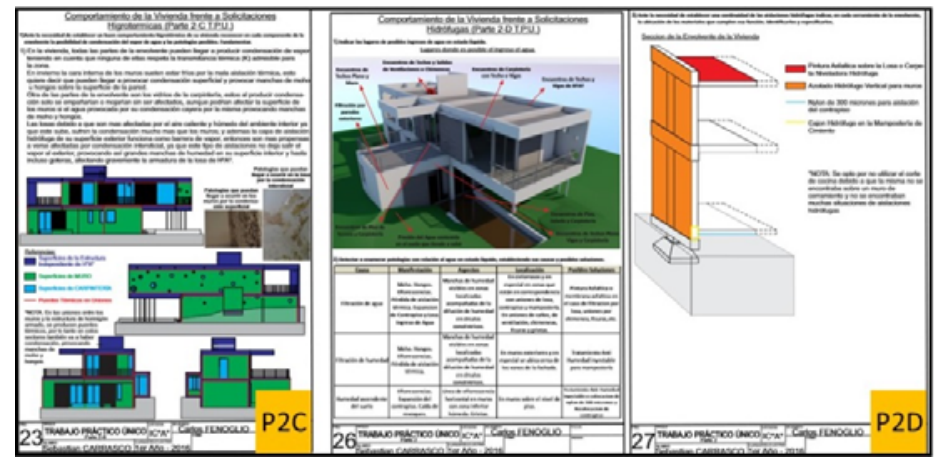

Fig. 6. Parte 2C y 2D Propiedades Higrotérmicas e Hidrófugas

En relación a las Propiedades Hidrófugas, en la Parte 2D, y a partir de un corte de techo a cimiento, el estudiante identifica los posibles ingresos de agua, determina sus patologías y enuncia los materiales utilizados como Aislantes Hidrófugos con sus características. Resulta importante tener en cuenta las uniones de las partes y las distintos tipos de subsistemas en una misma vivienda (construcción en seco o húmedo). Figura 6, parte P2D. Entender que el ingreso de agua en estado líquido puede ser perjudicial para el confort de nuestras viviendas y que las soluciones deben idearse en la etapa de diseño y controlarse en obra es parte de los objetivos de estas propiedades en esta Parte del trabajo.

Desarrollar destrezas y habilidades para comprender la importancia de la relación de los materiales, sus masas y sus superficies en la aislación acústica de un cerramiento vertical y comprender la importancia de un análisis integral de dichas propiedades, son objetivos destacados de la parte $2 \mathrm{E}$ Propiedades Acústicas. Tomando como base su dormitorio o estar (Figura 7) que dé al exterior, el estudiante realiza un análisis integral Acústico de su vivienda. A partir de un corte que muestre el interior del muro, identifica los materiales y sus espesores, calcula la masa de los mismos para luego a partir del grafico de Ley de Masas, determinar su Aislación en $1 \mathrm{~m} 2$. Mediante un esquema 
superficial del Cerramiento vertical, determina las superficies intervinientes, para luego con ese dato y la aislación de las partes, ingresa al gráfico de monograma para el cálculo de aislación acústica por particiones mixtas y determina la aislación total del cerramiento.

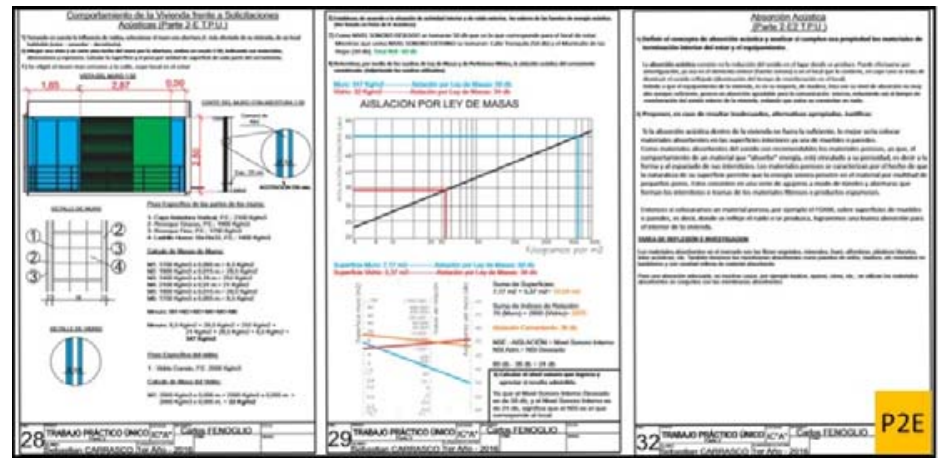

Fig. 7. Parte 2E. Propiedades Acústicas.

Teniendo en cuenta el Nivel Sonoro Externo (NSE) y la Aislación calculada, determina el Nivel Sonoro Interno (NSI) que deberá ser inferior o igual al Nivel Sonoro Interno deseado (NSId). A partir de aquel valor el estudiante plantea las posibles soluciones a tener en cuenta para lograr disminuir ese NSI. La temática se completa con el análisis de los distintos materiales y/o elementos interiores del ambiente en relación a la absorción acústica.

La figura 8, refiere a la Parte 3 del TPU Documentación gráfica de la Vivienda. Unidades Temáticas intervinientes: Modos de Expresión. El estudiante a partir de una planta, dos cortes y 2 vistas en escala 1:50, una planta y corte de la cocina en escala 1:20, determina las cotas totales y parciales, cotas de nivel de piso, la ubicación del norte, los límites del terreno (LM/EM) y denomina cada uno de los locales. Identificar los planos como elementos necesarios para ejecutar una obra y sintetizarlos para un lectura clara serán parte de los objetivos en esta instancia.

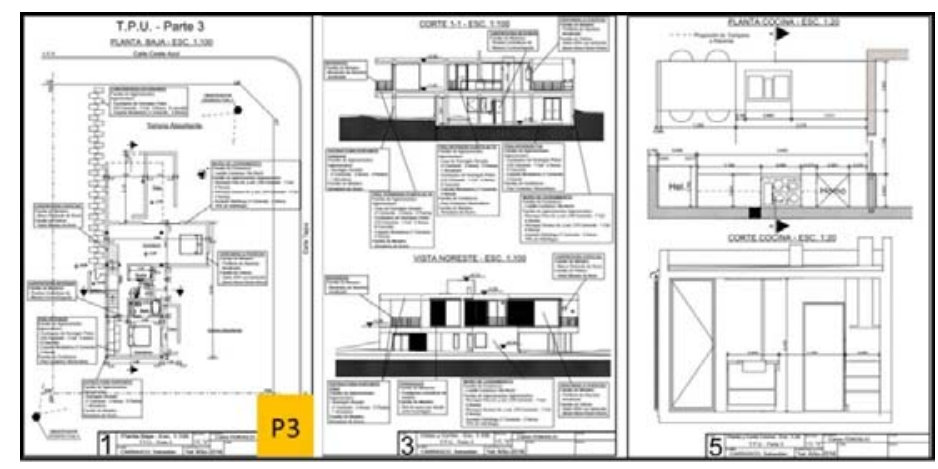

Fig. 8. Parte 3. Representación Gráfica.

La parte 4 del trabajo se refiere a las instalaciones. Comprende Instalaciones Sanitarias, Eléctricas y de Gas. En las Instalaciones Sanitarias debe identificar en una planta escala 1:50 y con colores convencionales, los elementos pertenecientes a los desagües primarios, secundarios y pluviales, las instalaciones de provisión de agua fría y agua caliente. En las Instalaciones Eléctricas, a partir de una planta similar a la anterior y con equipamiento fijo y móvil, identifica los elementos básicos de una instalación de estas características (bocas, tomacorrientes, llaves de encendido, Televisión, Internet, Teléfono, Tablero Primario o Tablero Secundario, Disyuntor, Llave Térmica, etc), y analiza si sus ubicaciones son las adecuadas. Figura 9. Para las Instalaciones de Gas y en planta escala 
1:50, el estudiante identifica cada uno de los artefactos, sus consumos y sus respectivas ventilaciones.

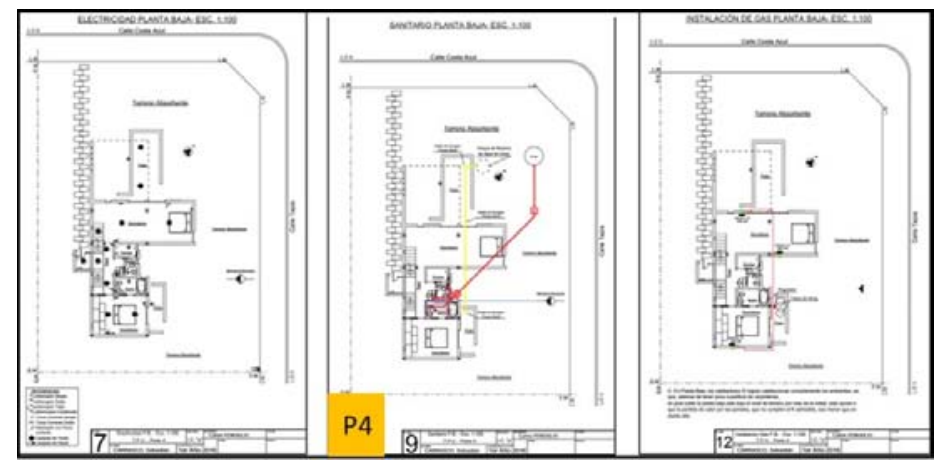

Fig. 9. Parte 4. Instalaciones

Entender que el subsistema instalaciones da respuesta a los requerimientos básicos para la habitabilidad y el confort humano, y que las mismas ocupan un espacio en su vivienda serán parte de los objetivos en esta instancia.

Identificar y analizar los materiales que conforman su vivienda. Interviniendo las Unidades Temáticas relacionadas con las familias de materiales y sus propiedades se identifican en la Parte 5 del TPU. A partir de una perspectiva axonométrica y/o corte, el estudiante señala la ubicación de los materiales de cada familia, detalla dimensiones y las tipifica, y reconoce su función dominante. Además determina sus características organolépticas, sus formas de comercialización, sus marcas comerciales y concluye si es adecuada su utilización en relación a su zona de origen. Figura 10.

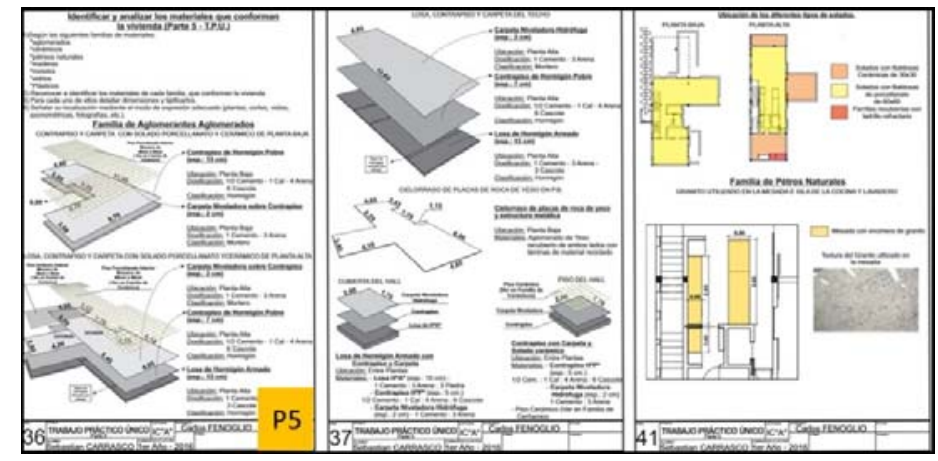

Fig. 10. Parte 5 TPU. Identificación de materiales

Del trabajo de Investigación que los estudiantes realizaron en equipos de hasta 4 alumnos, se desprende la parte 6 del TPU. En la misma desarrolla la investigación de un material con relación a un sub-sistema asignado por el docente. Dando respuesta a ese u otros sub-sistemas. Contiene como mínimo la siguiente información: Origen, materia prima/componentes de base o complementarios, grado de transformación, propiedades químicas-físicas-térmicas-acústicaseléctricas-mecánicas, características organolépticas, usos/localizaciones y tipos/variedad/marcas comerciales/centros de comercialización. Esta sección es acompañada con elementos de escritura, gráficos, esquemas, cuadros, y aquellos que considere necesarios. Es objetivo que el estudiante, a partir del análisis de las propiedades físico-químicas, las propiedades mecánicas y las propiedades tecnológicas, determine qué, cuánto y cómo utilizar un material. Figura 11. 


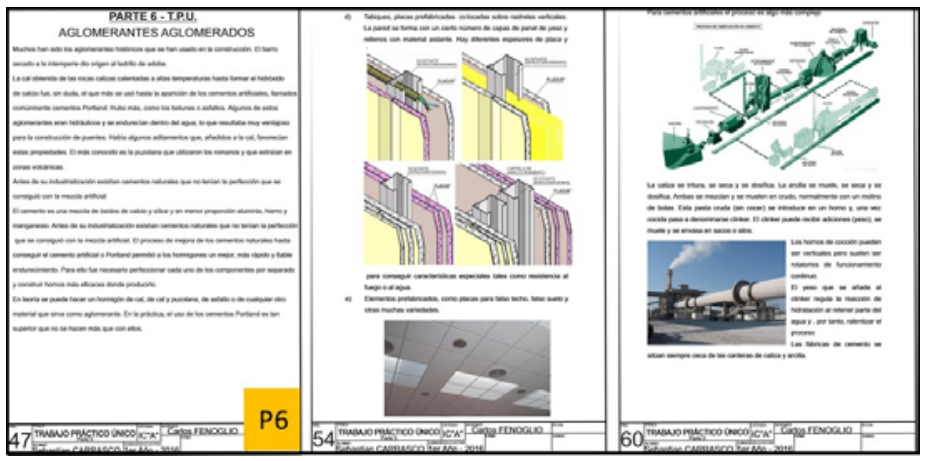

Fig. 11. Parte 6 TPU. Material Investigado

\section{EVALUACIÓN DEL TPU}

Al cierre de cada Unidad temática se realiza una corrección obligatoria de la sección referenciada del TPU que servirá de seguimiento y verificación de aquellos objetivos planteados.

La presentación del trabajo tiene dos etapas. La primera que comprende las partes 1 y 2 del mismo, se entrega y evalúa con una semana de antelación a la realización de la Práctica Evaluada Globalizadora $N^{\circ} 1$ (PEG) o primer parcial. La segunda etapa, con las partes 3, 4, 5 y 6 se entrega y evalúa previo a la realización de la segunda $\mathrm{PEG}$ o parcial.

EI TPU forma parte de las exigencias de aprobación de la asignatura junto a las Jornadas de Investigación, los dos parciales y el Ejercicio Integrador.

Es criterio general de la cátedra entender la evaluación como una instancia más del proceso enseñanza-aprendizaje. El proceso y el resultado son interdependientes y serán ponderados en todos los aspectos en el transcurso de la ejercitación.

La evaluación, es entendida desde el punto de vista del sujeto, permitiéndole utilizar al evaluador para consolidar o modificar su aprendizaje. Pero estamos inmersos en un sistema formal de aprendizaje con un compromiso académico (Universidad Pública) y social (ejercicio profesional), por ello las evaluaciones serán ponderadas con un número que representa un criterio de valoración.

Entre los aspectos básicos a evaluar destacamos: Vocabulario técnico; Conceptos terminológicos; Empleo de escalas y medios de expresión gráfica; Manejo de bibliografía; Relación entre los conocimientos adquiridos; Coherencia entre los distintos sistemas de comunicación.

Para la aprobación del TPU, el estudiante debe alcanzar cada uno de los objetivos propuestos de cada Unidad temática y la integración de las mismas.

\section{CONCLUSIONES}

Entendiendo la Arquitectura como hecho integral, a los efectos de su estudio, se propone como carera un Plan de Estudios con un abordaje por áreas de conocimiento con diferentes asignaturas, cada una de ellas, separadas en diferentes unidades temáticas. En este entorno se presenta como un desafío proponer situaciones didácticas que faciliten al estudiante, realizar la síntesis y asociación conceptual en su aprendizaje. 


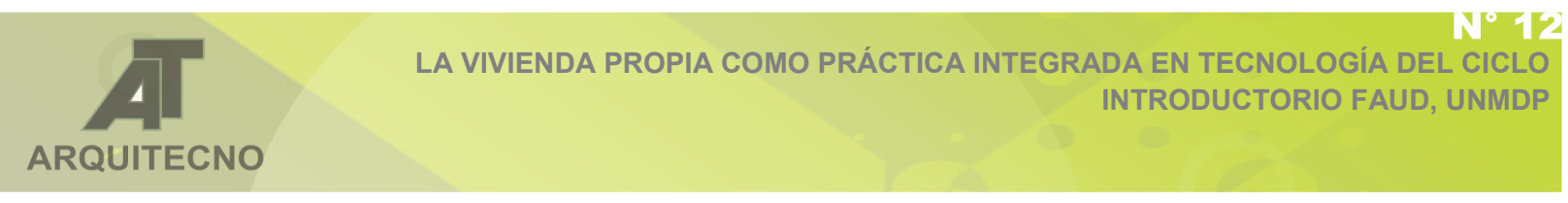

El trabajo Práctico Único (TPU) es un aporte para lograr la integración de aquellos saberes en contacto con la vivienda propia, conocida, vivida y experimentada por los estudiantes. Logrando una síntesis en los tres modos de abordaje al conocimiento realidad-iconización-modelización, que le permita interpretar y pasar de uno a otro con mayor facilidad.

Intenta ser base de la complejidad creciente, propia del avance académico general, y en particular del sub-área Construcciones, aportando elementos para la confección de la información técnica en la conceptualización y concreción de la obra arquitectónica.

A partir de las experiencias docentes, surge la necesidad de compartir los resultados, con la finalidad de incluir estos abordajes, en el ámbito de una formación profesional continua alimentando el espectro y amplitud de las acciones posibles.

\section{BIBLIOGRAFÍA}

Quaroni, L. (1980). Proyectar un edificio. Ocho lecciones de arquitectura. $3^{\circ}$ ed. Madrid (España). Xarait Ediciones.

Freire, P. (2012). Pedagogía de la autonomía. Saberes necesarios para la práctica educativa. $2^{\circ}$ ed. $4^{\circ}$ reimp. Buenos aires (Argentina). Ed. Siglo Veintiuno. 